\title{
The interrelationship of
}

\author{
HbA1c and real-time
}

\author{
continuous glucose
}

\section{monitoring in children with}

type 1 diabetes

Tamás Ferenci, Anna Körner, Levente

Kovács 


\section{Final publication}

DOI: http://dx.doi.org/10.1016/j.diabres.2015.01.019

(C) 2015, Elsevier. Licensed under the Creative Commons Attribution-

NonCommercial-NoDerivatives 4.0 International

http://creativecommons.org/licenses/by-nc-nd/4.0/

\section{(c)}




\section{Title page}

Title: The interrelationship of HbA1c and real-time continuous glucose monitoring in children with type 1 diabetes

Authors' names: Tamás Ferenci ${ }^{\mathrm{a}}$, Anna Körner $^{\mathrm{b}}$, Levente Kovács ${ }^{\mathrm{a}}$

Authors' affiliations: a: Óbuda University, John von Neumann Faculty of Informatics, Physiological Controls Group, Budapest, Hungary, H-1034

Bécsi út 96/b b: Semmelweis University, $1^{\text {st }}$ Department of Paediatrics, Budapest, Hungary, H-1083 Bókay János u. 53-54.

Authors’email addresses: Tamás Ferenci: ferenci.tamas@nik.uni-obuda.hu, Anna Körner: korner.anna@med.semmelweis-univ.hu, Levente Kovács: kovacs.levente@nik.uni-obuda.hu.

Corresponding author: name: Levente Kovács, address: H-1034 Budapest, Bécsi út 96/b, telephone number: 36 (1) 666-5585, fax number: 36 (1) 6665522, e-mail address: kovacs.levente@nik.uni-obuda.hu.

Article type: Original research article / Basic research.

Word count: 2058. 


\section{Abstract}

Aims: The aim of this observational study is to investigate the relationship between age, duration of diabetes, $\mathrm{HbA} 1 \mathrm{c}$ and the parameters of glucose levels measured with real-time CGM in children with type 1 diabetes.

Methods: Glucose level was characterized with the relative time spent in hyper- and hypoglycemia, central tendency, variability and MAGE during (real-time) CGM. These parameters were measured in 57 children with type 1 diabetes mellitus. The univariate association of the measured parameters was investigated with scatterplots as well as with linear and distance correlation coefficients.

Results: Age and duration of diabetes was not clinically relevantly associated with any descriptor of glucose level. HbA1c had an overall positive association with variability and MAGE observed during CGM. Slight, but non-significant, positive association of HbA1c was observed with the time spent in hyperglycemia and the central tendency of glucose level. With the exception of MAGE, the associations of the descriptors with HbA1c are non-monotonic, with a temporary break in the positive correlation at $10 \%$.

Conclusions: The results confirmed the well-known positive association of HbA1c with the central tendency of glucose level. The non-monotonic relationship between $\mathrm{HbA} 1 \mathrm{c}$ and the indicators of the central tendency of 
glucose level might be caused by the varying adherence of the patients during the CGM registration. HbA1c's positive association with MAGE without that non-monotonicity underlines MAGE's usefulness in the reliable assessment of the patients' glycemic state. 


\section{Keywords}

Pediatric type 1 diabetes mellitus; continuous glucose monitoring; glucose variability; Hawthorne effect; HbA1c. 


\section{Introduction}

Type 1 diabetes is one of the most common chronic disorders in children and adolescents. Several factors play a role in the pathogenesis of the complications in diabetes. Improved metabolic control in particular might prevent or postpone late vascular complications, as has been demonstrated by many studies, mainly by the Diabetes Control and Complications Trial [1] as well as by Epidemiology of Diabetes Interventions and Complications [2]. Glycemic control is usually determined by measurement of glycated hemoglobin (HbA1c). However, patients with similar glycated hemoglobin levels and mean glucose values can experience different glycemic fluctuations. Evidence implicates glycemic variability, mostly present in the form of postprandial glycemic spikes, as a key factor in the development of the complications of diabetes [3].

New technological improvements, especially continuous glucose monitoring (CGM) technique, which consists of a disposable transcutaneous glucose sensor along with an electronic transmitter/receiver unit [4], allows a more precise follow-up of the glucose evolution in vivo. Monitoring might be blinded to the patient which is used to inform healthcare professionals about the patient's glucose evolution, or non-blinded, also called personal or realtime CGM (RT-CGM), where the receiver is supplemented with a graphical display which allows the patients to have a direct, almost minute-to-minute 
observation of their own glucose level, thus enabling a better management of their diabetes. This has demonstrated to improve glucose control [5-6]. By their 5 minutes measurement frequency (288 measurements per day) the possibilities of CGM have direct effect on short term monitoring, but questions are raised on its usefulness for evaluating long-term outcome. The most important classical indicator in this sense is $\mathrm{HbAlc}$ that reflects the average glucose level of a patient over 120 days (the "life cycle" of red blood cells) [7]. Beside HbA1c, glycemic variability is now suggested to play role in the appearance of long-term complications of diabetes [8-9]. Correlation of HbAlc and glucose itself is well known, but CGM opened new horizons in a more thorough investigation of this issue [10-12]. The aim of the current work is to evaluate the relationship between age, duration of diabetes and HbA1c on the one hand, and glycemia measured with CGM on the other hand in children with type 1 diabetes. 


\section{Material and methods}

\subsection{Study population and design}

Fifty seven children with type 1 diabetes mellitus (DM) were selected as a convenience sample from the database of the $1^{\text {st }}$ Department of Paediatrics of Semmelweis University (Budapest, Hungary), with CGM measurement data between July 2009 and February 2011. Only patients with at least 1 year of DM duration were included so that even $\mathrm{HbA1c}$ had time to reach its characteristic levels.

The vast majority of the patient were poorly controlled (according to the guidelines [13] of the American Diabetes Association and the International Society for Pediatric and Adolescent Diabetes [14-16]) with CGM performed to optimize therapy. CGM measurements were non-blinded to the patients, i.e. real time (RT)-CGM was employed.

The sample included patients using continuous subcutaneous insulin infusion (CSII) and multiple daily injections (MDI) treatment regimes. The CGM sensors were accurate and reliable, time to sensor failure, frequency and duration of data gaps were negligible, the alarms worked properly. Sex, age, duration of diabetes and $\mathrm{HbA} 1 \mathrm{c}$ at the time of the CGM measurement were extracted for each patient from the electronic records of the hospital 
information system. HbA1c was measured with NGSP certified method. The summary of these patient characteristics is shown in Table 1.

CGM recordings were electronically processed (by exporting them to comma separated values format using the software of the device manufacturer) and the following parameters were extracted from the time series of glucose measurements to characterize the clinically relevant aspects of the patients' glucose evolution:

- Time spent in hyperglycemia and hypoglycemia relative to the whole length of recording (hypoglycemia was defined as glucose level $<3.9 \mathrm{mmol} / \mathrm{l}$, hyperglycemia was defined as glucose level $>10$ $\mathrm{mmol} / \mathrm{l})$ in $[\%]$.

- Central tendency of glucose levels (quantified with mean and median glucose level) in [mmol/l] .

- Dispersion, i.e. variability of glucose levels (quantified with standard deviation and interquartile range of glucose level) in [mmol/l].

- Glycemic variability as measured by Mean Amplitude of Glycemic Excursions (MAGE), calculated according to the algorithm of Baghurst [17-18]. While there are many glycemic variability metrics described in the literature [19], MAGE was now chosen due to its very widespread use even in spite of its limitations [20], i.e. to show 
a representative example of glycemic variability metrics to illustrate their application. Comparison of these metrics is extensively covered in the literature [21].

Summary of these parameters is presented in Table 2.

\subsection{Statistical analysis}

A pairwise analysis was performed between every possible variable (altogether $3 \cdot 7=21$ pairs). As every variable was quantitative, this was essentially a question of correlation, which was investigated with graphical (scatter plot) and analytical (calculation of correlation coefficients) methods.

Scatter plots sometimes revealed not simply non-linear, but markedly nonmonotonic relationships, so the calculation of the linear correlation coefficients is not sufficient. Spearman- $\rho$ correlation coefficient is often applied in situations where non-linearity is encountered [22], however it also falls short in detecting non-monotonic connections. Therefore, a method is needed that characterizes the overall dependence of two variables; now a modern metric for this purpose, called distance correlation [23-25] was used. Distance correlation is based on the difference between the joint probability density function of the variables and the product of their marginal density functions (as these should be equal if the variables are independent, hence, their difference characterizes the dependence of the variables). This way, distance correlation does not depend on the nature of 
the relationship, it can detect the strength of linear and non-linear, monotonic and non-monotonic, i.e. arbitrary connection between the variables.

Also to facilitate the detection of more complex connections, LOWESSestimators [26] for the non-parametric regression between the investigated variables were plotted on the scatter plots. Second-degree polynomial were used with a smoothing parameter of 0.75 . Ninety-five percent confidence intervals for the regression are also shown. These non-parametric regressions - and their confidence intervals - help visualizing the relationship between the investigated variables (without confining ourselves to any pre-specified function form of the relationship).

Due to the relatively low sample size, we did not attempt to perform an agestratified analysis, to maintain the reliability of the results.

Statistical analysis and visualization was performed under the R statistical environment, version 3.0.2 [27]. The R script developed for this purpose is available at the corresponding author on request. 


\section{Results}

Age and duration of DM showed no clear relationship with any descriptor of the glucose levels as evidenced by the correlation coefficients (Table 3 ) and the scatterplots (figures not shown here).

HbA1c (Figure 1) however shows a clear - but statistically non-significant overall positive association with ratio of time spent in hyperglycemia (but not with hypoglycemia). Note that a small, seemingly paradoxical nonmonotonic region at about $\mathrm{HbA} 1 \mathrm{c}=10 \%$ can be observed. As far as the central tendency is concerned, again a clear, but statistically non-significant overall positive association can be observed both for mean and median. The paradoxical non-monotonic region around 10\% shows up again. However, for glycemic variability, there is a clear and statistically significant positive association (both for standard deviation and interquartile range). The trend change about $10 \%$ is not as pronounced as before; rather, a flat region can be seen on the scatter plots.

MAGE (Figure 2) only has a slight positive association with HbA1c (and the paradoxical region around $10 \%$ is missing, too).

Linear correlation coefficients are significant for glucose variability vs. HbA1c $(p<0.005)$ and MAGE vs. HbA1c $(p=0.0385)$. Time spent in hyperglycemia is at the border of significance. Distance correlations are significant likewise for variabilities $(p=0.0165$ and $p=0.0063)$, and is at the 
border of significance for MAGE $(p=0.0676)$. Note that distance correlation is always between 0 and 1, as there is no point in talking about the direction of the relationship for a non-monotonic function. The $p$-values of the distance correlations are expected to be higher, given the fact that this test has lower power (due to its more general nature). 


\section{Discussion}

Duration of diabetes and age is not associated with any indicator of glucose evolution during CGM in clinically relevant way. On the other hand, HbA1c is positively - and statistically significantly - associated with the indicators of the variability of glucose levels and MAGE. Compared to the duration of diabetes and age, stronger and clinically relevant, but statistically still nonsignificant, overall positive association can be observed between $\mathrm{HbA} 1 \mathrm{c}$ and the indicators of the central tendency of glucose level and time spent in hyperglycemia. (Non-significance of these is likely attributable to the low sample size.) These associations - especially for the time spent in hyperglycemia and the central tendency of glucose levels - are not linear, furthermore not even monotonic, as they show a marked temporary decrease around $\mathrm{HbA} 1 \mathrm{c}=10 \%$.

Note that as now 21 hypothesis testings were performed in parallel (one for each patient characteristic/CGM-property combination), results cannot be compared to the traditional threshold of $5 \%$ due to the multiple comparisons situation [28]. However, significant correlations - especially for variability - had magnitude smaller $p$-values, so labeling of these as significant association is justified, even in the light of the multiple comparisons situation. 
The positive association between the indicators of the central tendency of glucose and HbA1c is well known, even quantitatively [29-31].

Positive association between the indicators of the variability and HbA1c are also described in the literature [32] but not with uniform results [33-34].

Our results also confirm the suggested [34] association between hyperglycemia and $\mathrm{HbA} 1 \mathrm{c}$.

The explanation for the seemingly paradoxical behavior of the association of $\mathrm{HbA1c}$ with the various descriptors of glucose levels is possibly the poor adherence that is characteristic for most of the investigated children. The motivation to "cheat" during the CGM-measurements (i.e. to pay closer attention to glucose levels than it is usually done by the children) is higher for those having worse metabolic state (as evidenced by HbA1c higher than $8 \%$ ). Also, there is not only a motivation, but also a possibility for this, given that the measurements were non-blinded for the children. Hence, it may be hypothesized that children above $8 \% \mathrm{HbA} 1 \mathrm{c}$ start to increasingly pay unusual - i.e. that is not done without CGM - attention to their glucose levels during CGM measurement, breaking the association between $\mathrm{HbA1c}$ and the mean (or median) glucose during CGM. They could, however, do it only up to a point (about 10\%) as even worse metabolic states could not be “compensated" by such temporarily increased adherence. This can be more directly illustrated when $\mathrm{HbA} 1 \mathrm{c}$ is converted to (long-term) estimated 
average glucose and is contrasted with the average glucose (i.e. short-term average) that can be calculated from the CGM recording (Figure 3). The conversion of $\mathrm{HbA} 1 \mathrm{c}$ to estimated average glucose was done using the formula $e A G_{m m o l / l}=1.59 \times H b A 1 c \%-2.59$ [29].

This phenomenon is the well-known Hawthorne effect studied in many fields of science, and already described in relationship to CGM as well [35]. Hawthorne effect claims that patients - not necessarily deliberately - alter their behavior when they know that they are under observation.

The small sample size and the convenience sampling of the present study limits the robustness of this conclusion, and further studies are needed to confirm this finding, and - if confirmed - investigate its determinants. 


\section{Conclusion}

The results confirmed the well-known positive association of $\mathrm{HbA} 1 \mathrm{c}$ with the indicators of the central tendency of glucose (mean, median). No association was found between the duration of the diabetes and the age of the patient with any indicator of the glucose levels.

The relationship between HbA1c and the indicators of the central tendency of glucose are non-monotonic, which is likely caused by the varying adherence of the patients. HbA1c was also positively associated with MAGE, but the non-monotonic region was missing, underlining MAGE's usefulness in the reliable assessment of the patients' glycemic state. 


\section{Acknowledgments}

Levente Kovács is supported by the János Bolyai Research Scholarship of the Hungarian Academy of Sciences. 


\section{Author disclosure statement}

The authors declare that they have no conflict of interest. 


\section{Authors' contribution}

TF performed the statistical analysis and drafted the manuscript. AK conceived the research and provided support in clinical interpretations. LK supervised the project and revised the manuscript. All authors have read and approved the final article. 


\section{Role of the funding source}

No specific funding was used, neither to conduct the research nor to prepare the article. 


\section{References}

1. The Diabetes Control and Complications Trial Research Group: The Effect of Intensive Treatment of Diabetes on the Development and Progression of Long-Term Complications in Insulin-Dependent Diabetes Mellitus. N Engl J Med 1993;329:977-986. DOI: 10.1056/NEJM199309303291401.

2. Gubitosi-Klug RA; DCCT/EDIC Research Group. The diabetes control and complications trial/epidemiology of diabetes interventions and complications study at 30 years: summary and future directions. Diabetes Care 2014; 37:44-49. DOI: $10.2337 / \mathrm{dc} 13-2148$.

3. Monnier L, Colette C, Owens DR: Glycemic variability: the third component of the dysglycemia in diabetes. Is it important? How to measure it? Diabetes Sci Technol 2008;2:1094-1100. DOI: $10.1177 / 193229680800200618$.

4. Wolpert HA: Continuous glucose monitoring-coming of age. $\mathrm{N}$ Engl J Med 2010;363:383-384. DOI: 10.1056/NEJMe1006098.

5. Szypowska A, Ramotowska A, Dzygalo K et al: Beneficial effect of real-time continuous glucose monitoring system on glycemic control in type 1 diabetic patients: systematic review and meta-analysis of 
randomized trials. Eur J Endocrinol 2012;166:567-574. DOI:

10.1530/EJE-11-0642.

6. Leinung M, Thompson S, Nardacci E: Benefits of continuous glucose monitor use in clinical practice. Endocr Pract 2010;16:371375. DOI: 10.1089/dia.2013.0027.

7. LeRoith D, Taylor SI, Olefsky JM: Diabetes Mellitus. A fundamental and clinical text. Philadelphia, Lippincott Williams \& Wilkinson, 2004.

8. Hirsch IB, Brownlee M: Should minimal blood glucose variability become the gold standard of glycemic control? J Diabetes Complications 2005;19:178-181. DOI:

10.1016/j.jdiacomp.2004.10.001.

9. Thomas A, Schönauer M, Achermann F, et al: The "glucose pentagon": assessing glycemic control of patients with diabetes mellitus by a model integrating different parameters from glucose profiles. Diabetes Techn Ther 2009;11:399-409. DOI: 10.1089/dia.2008.0119.

10. Nielsen JK, Gravholt CH, Djurhuus CB, et al: Continuous Subcutaneous Glucose Monitoring Shows a Close Correlation between Mean Glucose and Time Spent in Hyperglycemia and 
Hemoglobin A1c. J Diabetes Sci Technol 2007;1:857-863. DOI: 10.1177/193229680700100609.

11. Mazze R: The Future of Self-Monitored Blood Glucose: Mean Blood Glucose Versus Glycosylated Hemoglobin. Diabetes Techn Ther 2008;10:S93-S101. DOI: 10.1089/dia.2008.0006.

12. Sikaris K. The Correlation of Hemoglobin A1c to Blood Glucose. J Diabetes Sci Technol 2009 May 1;3(3):429-438. DOI: 10.1177/193229680900300305.

13. Wood JR, Miller KM, Maahs DM, et al. Most youth with type 1 diabetes in the T1D Exchange Clinic Registry do not meet American Diabetes Association or International Society for Pediatric and Adolescent Diabetes clinical guidelines. Diabetes Care 2013 Jul;36(7):2035-2037. DOI: 10.2337/dc12-1959.

14. Acerini C, Craig ME, de Beaufort C, Maahs DM, Hanas R. Introduction to ISPAD Clinical Practice Consensus Guidelines 2014 Compendium. Pediatr Diabetes 2014;15(Suppl 20):1-3. DOI: 10.1111/pedi.12182.

15. American Diabetes Association. Standards of medical care in diabetes - 2014. Diabetes Care 2014;37(Suppl. 1):S14-S80. DOI: 10.2337/dc14-S014. 
16. Donaghue KC, Chiarelli F, Trotta D, Allgrove J, Dahl-Jorgensen K, International Society for Pediatric and Adolescent Diabetes. ISPAD clinical practice consensus guidelines 2006-2007. Microvascular and macrovascular complications. Pediatr Diabetes 2007;8:163-170.

DOI: 10.1111/j.1399-5448.2007.00250.x.

17. Service FJ, Molnar GD, Rosevear JW, et al: Mean amplitude of glycemic excursions, a measure of diabetic instability. Diabetes 1970;19:644-655. DOI: 10.2337/diab.19.9.644.

18. Baghurst PA: Calculating the mean amplitude of glycemic excursion from continuous glucose monitoring data: an automated algorithm. Diabetes Techn Ther 2011;13:296-302. DOI: 10.1089/dia.2010.0090.

19. Cameron FJ, Baghurst PA, Rodbard D. Assessing glycemic variation: why, when and how? Pediatr Endocrinol Rev 2010; Suppl $3: 432-444$.

20. Cameron FJ, Donath SM, Baghurst PA. Measuring glycaemic variation. Curr Diabetes Rev 2010; 6:17-26. DOI: $10.2174 / 157339910790442592$. 
21. Rodboard D. Interpretation of continuous glucose monitoring data: glycemic variability and quality of glycemic control. Diabetes Technol Ther 2009; Suppl 1:S55-67. DOI: 10.1089/dia.2008.0132.

22. Hollander M, Wolfe DA: Nonparametric statistical methods. New York, Wiley, 1973.

23. Székely GJ, Rizzo ML, Bakirov NK: Measuring and testing independence by correlation of distances. Ann Stat 2007;35:27692794. DOI: $10.1214 / 009053607000000505$.

24. Székely GJ, Rizzo ML: Energy statistics: A class of statistics based on distances. J Stat Plan Inference 2013;143:1249-1272. DOI: 10.1016/j.jspi.2013.03.018.

25. Székely GJ, Rizzo ML: Partial Distance Correlation with Methods for Dissimilarities. Ann Stat 2014;42:2382-2412. DOI: 10.1214/14AOS1255.

26. Cleveland WS, Grosse E Shyu WM: Local regression models, Chamber SJM, Hastie TJ (eds.) Statistical Models in S, Wadsworth \& Brooks/Cole, 1992.

27. R Core Team: R, A language and environment for statistical computing, R Foundation for Statistical Computing, Vienna, Austria, 2013. 
28. Miller RG: Simultaneous Statistical Inference, New York, Springer, 1981.

29. Nathan DM, Kuenen J, Borg R, et al: Translating the A1C Assay Into Estimated Average Glucose Values. Diabetes Care 2008;31:1473-1478. DOI: 10.2337/dc08-0545.

30. Makris I, Spanou L: Is There a Relationship between Mean Blood Glucose and Glycated Hemoglobin? J Diabetes Sci Technol 2011;5:1572-1583. DOI: 10.1177/193229681100500634.

31. Sacks DB: Correlation between Hemoglobin A1c (HbA1c) and Average Blood Glucose: Can HbA1c Be Reported as Estimated Blood Glucose Concentration? J Diabetes Sci Technol 2007;1:801803. DOI: $10.1177 / 193229680700100602$.

32. McCarter RJ, Hempe JM, Chalew SA: Mean Blood Glucose and Biological Variation Have Greater Influence on HbA1c Levels Than Glucose Instability An analysis of data from the Diabetes Control and Complications Trial. Diabetes Care, 2006;29(2), 352-355. DOI: 10.2337/diacare.29.02.06.dc05-1594.

33. Jin SM, Kim TH, Bae JC, et al: Clinical factors associated with absolute and relative measures of glycemic variability determined by continuous glucose monitoring: an analysis of 480 subjects. Diabetes 
Res Clin Pract, 2014; 104(2):266-272. DOI:

10.1016/j.diabres.2014.02.003.

34. Kohnert KD, Augstein P, Heinke P, Zander E, Peterson K, Freyse

EJ, Salzsieder E: Chronic hyperglycemia but not glucose variability determines HbA1c levels in well-controlled patients with type 2 diabetes. Diabetes Res Clin Pract, 2007; 77(3), 420-426. DOI: 10.1016/j.diabres.2007.01.021.

35. Fabiato K, Buse J, Duclos M, et al: Clinical experience with continuous glucose monitoring in adults. Diabetes Techn Ther 2009;11:S93-S103. DOI: 10.1089/dia.2009.0004. 


\section{Figure captions}

\section{Figure 1}

Title: Association of hyper- and hypoglycemia, central tendency and dispersion during CGM with $\mathrm{HbA1c}$.

Description: Scatterplots of HbA1c and the various descriptors of the CGMmeasured glucose profile. Solid line indicates the best fitting non-parametric regression curve obtained with LOWESS, gray shading shows $95 \%$ confidence interval for this curve. 

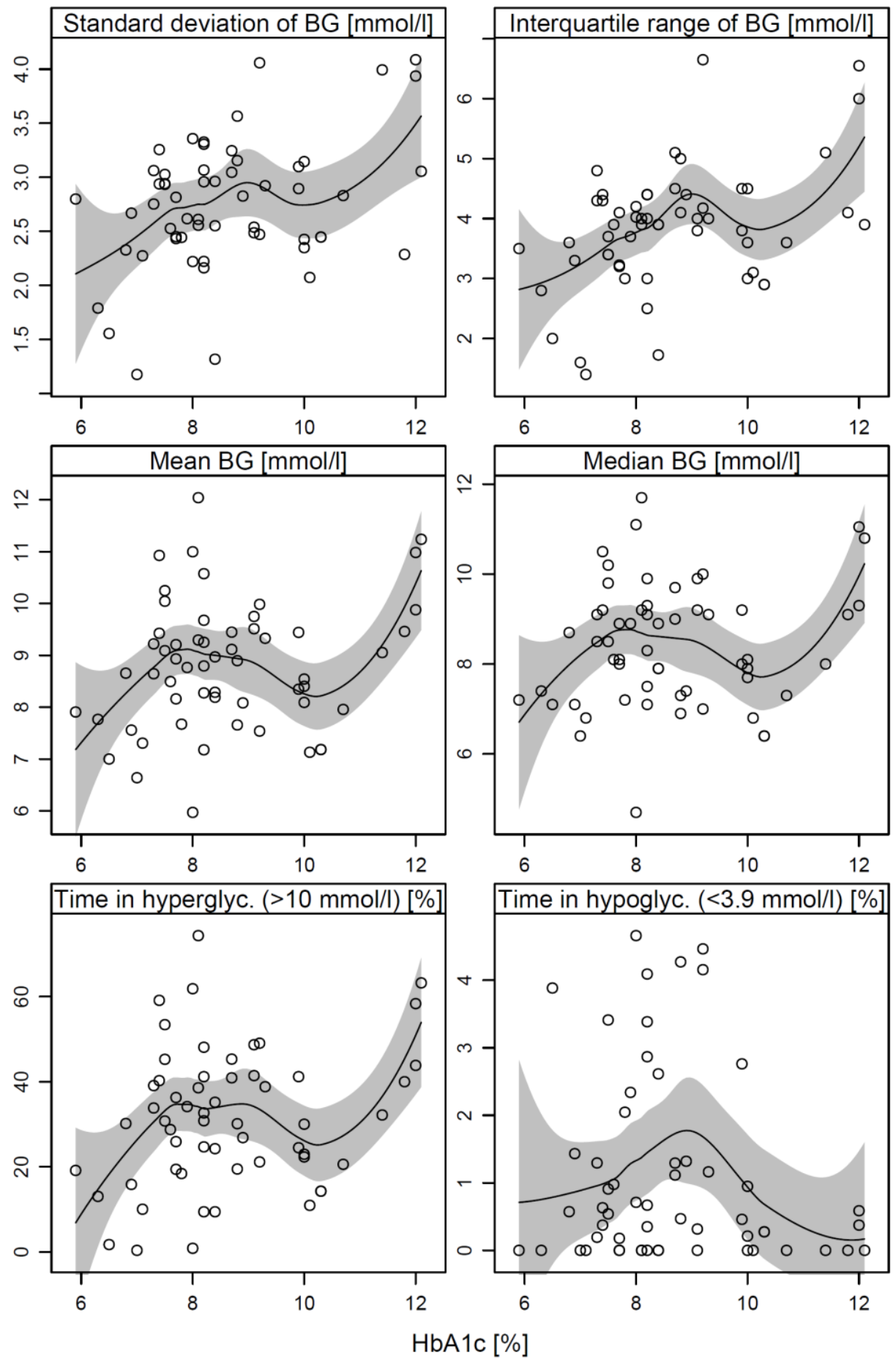


\section{Figure 2}

Title: Association of MAGE during CGM with HbA1c.

Description: Scatterplot of HbA1c and MAGE of the CGM-measured glucose profile. Solid line indicates the best fitting non-parametric regression curve obtained with LOWESS, gray shading shows $95 \%$ confidence interval for this curve.

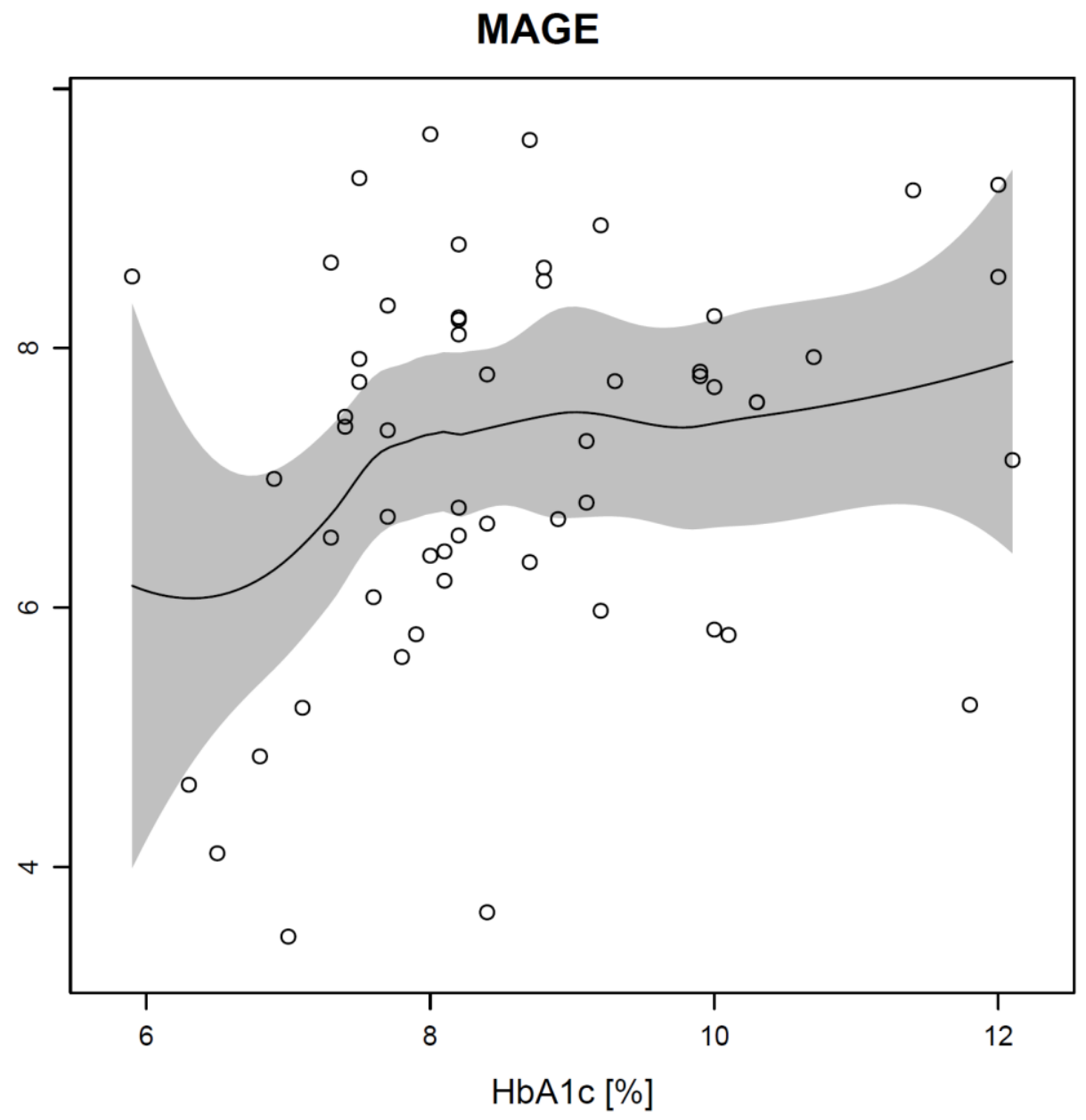




\section{Figure 3}

Title: Estimated average glucose calculated from $\mathrm{HbA1c}$ (i.e. long-term average) vs. average glucose during CGM (i.e. short-term average).

Description: Scatterplot of estimated average glucose calculated from HbA1c and average glucose during CGM. Dashed line indicates the best fitting non-parametric regression curve obtained with LOWESS, gray shading shows $95 \%$ confidence interval for this curve. Solid line is the reference curve indicating the equality of the two types of average glucose values. 


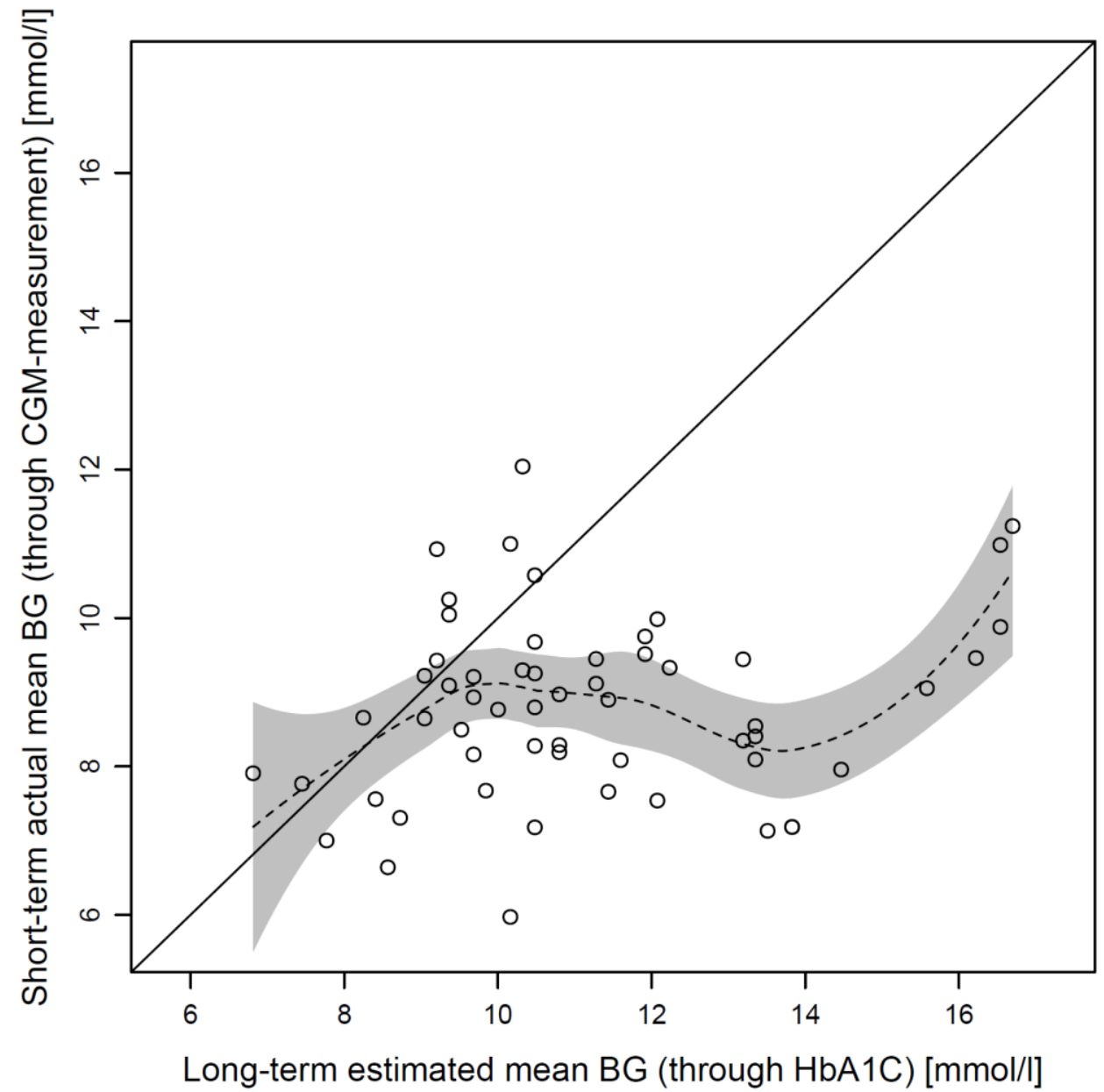




\section{Tables}

\section{Table 1}

Title: Most important descriptive statistics of the patients' characteristics.

Description: Patient characteristics in Mean (Median) $\pm S D(I Q R)$ [Min-

Max] format for continuous variables and as frequency tables for discrete variables.

\begin{tabular}{|l|l|}
\hline \multicolumn{1}{|c|}{ Parameter } & \multicolumn{1}{|c|}{ Descriptive statistics } \\
\hline Treatal number of patients & $30(52.6 \%)$ MDI, $27(47.4 \%)$ insulin pump \\
Length of CGM measurement [hours] & $216.9(161.6) \pm 166.7(258.1)[19.5-705.5]$ \\
Type of device & $47(82.5 \%)$ Guardian REAL-Time \\
& $9(15.8 \%)$ Paradigm 522 and 722 \\
Sex & $1(1.8 \%)$ Paradigm Veo-554 \\
Age [year] & $36(63.2 \%)$ female, 21 (36.8\%) male \\
Duration of DM [year] & $12.5(13.0) \pm 3.5(6.0)[4-18]$ \\
HbA1c [\%] & $5.1(4.0) \pm 3.5(6.0)[1-18]$ \\
HbA1c [mmol/mol] & $8.6(8.2) \pm 1.5(1.7)[5.9-12.1]$ \\
& $70.8(66.1) \pm 16.1(18.6)[41.0-108]$ \\
\hline
\end{tabular}




\section{Table 2}

Title: Most important descriptive statistics of the investigated CGM parameters.

Description: Descriptive statistics of the indicators derived from CGMmeasured glucose evolution in Mean (Median) $\pm S D$ (IQR) [Min-Max] format.

\begin{tabular}{|l|c|}
\hline \multicolumn{1}{|c|}{ Parameter } & Descriptive statistics \\
\hline Percentage of time spent in hyperglycemia & $31.3(30.8) \pm 16.3(21.7)[0.4-74.3]$ \\
Percentage of time spent in hypoglycemia & $1.1(0.5) \pm 1.4(1.3)[0-4.7]$ \\
$(<3.9 \mathrm{mml} / \mathrm{l})[\%]$ & $8.8(8.8) \pm 1.23(1.49)[5.97-12.04]$ \\
Mean glucose level [mmol/l] & $8.42(8.3) \pm 1.38(1.9)[4.7-11.7]$ \\
Median glucose level [mmol/l] & $2.75(2.8) \pm 0.59(0.62)[1.18-4.09]$ \\
Glucose level standard dev. [mmol/l] & $3.85(3.9) \pm 1.01(1.1)[1.4-6.65]$ \\
Glucose level IQR [mmol/l] & $7.17(7.39) \pm 1.47(1.89)[3.46-9.65]$ \\
MAGE & \\
\hline
\end{tabular}




\section{Table 3}

Title: Correlation of the investigated parameters.

Description: Asymmetric correlation matrix of the investigated parameters (linear and distance correlation coefficients with significance).

\begin{tabular}{|c|c|c|c|c|c|c|c|}
\hline & \multicolumn{3}{|c|}{ Linear correlation } & \multicolumn{3}{|c|}{ Distance correlation } \\
\hline & & $\begin{array}{c}\text { Age } \\
\text { [year] }\end{array}$ & $\begin{array}{c}\text { DM duration } \\
\text { [year] }\end{array}$ & $\begin{array}{c}\text { HbA1c } \\
{[\%]}\end{array}$ & $\begin{array}{c}\text { Age } \\
\text { [year] }\end{array}$ & $\begin{array}{c}\text { DM duration } \\
\text { [year] }\end{array}$ & $\begin{array}{c}\text { HbA1c } \\
{[\%]}\end{array}$ \\
\hline \multirow{2}{*}{ Ratio of hyperglycemia (>10 mmol/l) [\%] } & $r$ & 0.04 & -0.01 & 0.24 & 0.21 & 0.18 & 0.27 \\
\hline & $p$ & 0.7403 & 0.9514 & 0.0775 & 0.5540 & 0.7953 & 0.1642 \\
\hline \multirow{2}{*}{ Ratio of hypoglycemia $(<3.9 \mathrm{mml} / \mathrm{l})[\%]$} & $r$ & -0.04 & 0.02 & -0.14 & 0.15 & 0.18 & 0.24 \\
\hline & $p$ & 0.7801 & 0.8723 & 0.2871 & 0.8881 & 0.5777 & 0.1929 \\
\hline \multirow{2}{*}{ Mean glucose level [mmol/l] } & $r$ & 0.05 & 0.03 & 0.21 & 0.22 & 0.20 & 0.26 \\
\hline & $p$ & 0.7226 & 0.8291 & 0.1200 & 0.4637 & 0.6394 & 0.1904 \\
\hline \multirow{2}{*}{ Median glucose level [mmol/l] } & $r$ & 0.02 & 0.04 & 0.14 & 0.20 & 0.20 & 0.23 \\
\hline & $p$ & 0.8567 & 0.7689 & 0.2855 & 0.6163 & 0.5904 & 0.3482 \\
\hline \multirow{2}{*}{ Glucose level standard deviation [mmol/l] } & $r$ & 0.13 & -0.15 & 0.38 & 0.22 & 0.22 & 0.36 \\
\hline & $p$ & 0.3467 & 0.2730 & 0.0032 & 0.4869 & 0.4757 & 0.0165 \\
\hline \multirow{2}{*}{ Glucose level IQR [mmol/l] } & $r$ & 0.09 & -0.08 & 0.42 & 0.17 & 0.22 & 0.39 \\
\hline & $p$ & 0.4881 & 0.5497 & 0.0013 & 0.9606 & 0.5756 & 0.0063 \\
\hline \multirow{2}{*}{ MAGE } & $r$ & 0.10 & -0.17 & 0.27 & 0.19 & 0.25 & 0.30 \\
\hline & $p$ & 0.4727 & 0.1989 & 0.0385 & 0.7057 & 0.2390 & 0.0676 \\
\hline
\end{tabular}


